EPJ Web of Conferences 70, 00015 (2014)

DOI: 10.1051/epjconf/20147000015

(C) Owned by the authors, published by EDP Sciences, 2014

\title{
Proton-proton collisions at ultra-relativistic energies in quark- gluon string model
}

\author{
L. Bravina ${ }^{1, a}$, J. Bleibel ${ }^{2,3}$, L. Malinina ${ }^{4}$, M.S. Nilsson ${ }^{1}$, and E. Zabrodin ${ }^{1,4}$ \\ ${ }^{1}$ Department of Physics, University of Oslo, PB 1048 Blindern, N-0316 Oslo, Norway \\ ${ }^{2}$ Max-Planck-Institut für Intelligente Systeme, Heisenbergstr. 3, D-70569 Stuttgart, Germany \\ ${ }^{3}$ Institut für Augewandte Physik, Universität Tübingen, Auf der Morgenstelle 10, D-72076 Tübingen, Ger- \\ many \\ ${ }^{4}$ Skobeltsyn Institute of Nuclear Physics, Moscow State University, RU-119991 Moscow, Russia
}

\begin{abstract}
The microscopic Monte Carlo quark-gluon string model (QGSM) is employed to study particle production in ultrarelativistic proton-proton collisions. The model is based on Reggeon Field theory accomplished by string phenomenology. Various observables, including multiplicity, rapidity and transverse momentum spectra, short-range, long-range and femtoscopy correlations, are described quite well in a wide span of the collision energy. Predictions are made for $p p$ collisions at $\sqrt{s}=14 \mathrm{TeV}$.
\end{abstract}

\section{Introduction}

Despite the significant progress achieved in the last years, the theory of multiparticle production in elementary proton-proton collisions at ultrarelativistic energies is not completed yet. We all know, of course, that the processes with large momentum transfer $Q^{2}$ are well described by the perturbative chromodynamics (pQCD). Unfortunately, even at very high energies the main contribution to multiparticle production in hadronic interactions comes from the processes with small momentum transfer. This means that the running coupling constant $\alpha_{s}\left(Q^{2}\right)$ is not small and, therefore, the perturbative series expansion is not very helpful. Other techniques, based on non-perturbative methods, should be utilized. The quark-gluon string model (QGSM) [1] and similar to it dual parton model (DPM) [2] is one of the possible approaches to solution of this very interesting and difficult problem. Both models are based on the Reggeon Field theory (RFT) [3]. Basic principles of the QGSM are sketched below.

\section{The QGSM model}

The quark-gluon string model, formulated about 30 years ago [1], employs the $1 / N$ series expansion of the amplitude of a process in QCD, where $N$ is either the number of colors [4] or the number of flavors [5]. This method is also called topological expansion, because of emergence of diagrams of various topologies. Although it is not possible to assign weights for the diagrams within the QCD, there is one-to-one mapping between the diagrams in $1 / N$-expansion and the processes with exchange

\footnotetext{
ae-mail: larissa.bravina@fys.uio.no
} 
of Regge singularities in the $t$-channel. For instance, exchange of quantum numbers via Reggeons corresponds to planar diagrams, whereas the cylinder diagrams are represented by the reactions without the quantum number exchange. The latter proceed via the exchange of Pomerons. Therefore, the perturbative Reggeon Field Theory (RFT) [3] is directly linked to quantum chromodynamics.

The Monte Carlo version of the QGSM [6-8] employs statistical weights, hadron structure functions and leading quark fragmentation functions obtained from the Regge approach in [1] to choose subprocesses of string production, to compute mass and momentum of strings and to simulate string decays, respectively. The hadron-hadron collision part of the model includes single and double diffraction subprocesses, antibaryon-baryon annihilations and elastic scattering. The hadron inelastic interaction cross section $\sigma_{\text {inel }}(s)=\sigma_{\text {tot }}(s)-\sigma_{e l}(s)$ is split further into the cross section for single diffractive interactions $\sigma_{S D}(s)$ and the cross section for non-diffractive reactions $\sigma_{N D}(s)$, similar to analysis of experimental data. By means of the Abramovskii-Gribov-Kancheli (AGK) cutting rules [9] the inelastic non-diffractive interaction cross section $\sigma_{N D}(s)$ can be expressed via the sum of the cross sections for the production of $n=1,2, \ldots$ pairs of quark-gluon strings, or cut Pomerons, and the cross section of double diffractive process

$$
\sigma_{N D}(s)=\sum_{n=1}^{\infty} \sigma_{n}(s)+\sigma_{D D}(s) .
$$

To find $\sigma_{n}(s)$ one can utilize the quasi-eikonal model $[10,11]$ which states that

$$
\begin{aligned}
\sigma_{t o t}(s) & =\sum_{n=0}^{\infty} \sigma_{n}(s)=\sigma_{P} f\left(\frac{z}{2}\right), \\
\sigma_{n}(s) & =\frac{\sigma_{P}}{n z}\left(1-\exp (-z) \sum_{k=0}^{n-1} \frac{z^{k}}{k !}\right), k \geq 1 \\
\sigma_{0} & =\sigma_{P}\left(f\left(\frac{z}{2}\right)-f(z)\right), \\
f(z) & =\sum_{v=1}^{\infty} \frac{(-z)^{v-1}}{v v !},
\end{aligned}
$$

Here

$$
\begin{aligned}
\sigma_{P} & =8 \pi \gamma_{P} \exp (\Delta \xi) \\
z & =\frac{2 C \gamma_{P}}{\left(R_{P}^{2}+\alpha_{P}^{\prime} \xi\right)} \exp (\Delta \xi)
\end{aligned}
$$

The cross section $\sigma_{0}$ corresponds to diffraction contribution. The parameters $\gamma_{P}$ and $R_{P}$ are Pomeronnucleon vertex parameters, quantity $\Delta \equiv \alpha_{P}(0)-1$, and $\alpha_{P}(0)$ and $\alpha_{P}^{\prime}$ is the intercept and the slope of the Pomeron trajectory, respectively. The quantity $C$ takes into account the deviation from the pure eikonal approximation $(C=1)$ due to intermediate inelastic diffractive states, $\xi=\ln \left(s / s_{0}\right)$ and $s_{0}$ is a scale parameter.

The hard gluon-gluon scattering and semi-hard processes with quark and gluon interactions are also incorporated in the model, see [12]. For the modeling of string fragmentation the Field-Feynman algorithm [13] is employed. It enables one to consider emission of hadrons from both ends of the string with equal probabilities. The break-up procedure invokes the energy-momentum conservation and the preservation of the quark numbers.

Due to uncertainty principle it takes time to create a hadron from constituent quarks, e.g., fast particles are created the last. In string models two definitions of formation time are accepted [14]: 
the time when string is broken and all constituents of the hadron are created (constituent) or the time when the trajectories of hadron constituents (quarks) cross ("yo-yo"). In this version of QGSM we are using the constituent formation time. The formation time $t_{i}^{*}$ and coordinate $z_{i}^{*}$ of $i$-th hadron in the string center of mass can be expressed through its energy $E_{i}^{*}$, its longitudinal momentum $p_{z i}^{*}$ and the longitudinal momenta/energies of all hadrons produced by the decay of this string as

$$
t_{i}^{*}=\frac{1}{2 \kappa}\left(M_{s}-2 \sum_{j=1}^{i-1} p_{z j}^{*}\right), \quad z_{i}^{*}=\frac{1}{2 \kappa}\left(M_{s}-2 \sum_{j=1}^{i-1} E_{j}^{*}\right)
$$

Then we calculate $t_{i}$ in the laboratory frame and make the propagation of the coordinates to this point $\left(x_{i}, y_{i}, z_{i}, t_{i}\right): a_{i}=a_{0 i}+t_{i} p_{a i} / E_{i}, a=x, y, z$. Note, that $\kappa$ acts as a scaling parameter of the particle formation time.

\section{Results}

\subsection{Bulk observables and scaling relations}

Let us first consider bulk characteristics of particle production in $p p$ collisions at ultrarelativistic energies. Recall that at energies $\sqrt{s} \geq 50 \mathrm{GeV}$ the annihilation cross section is extremely small and, therefore, the main features of particle production in $p p$ interactions are similar to those in $\bar{p} p$ ones. Thus, for the comparison with the model results we utilized data obtained by the UA5 Collaboration for proton - antiproton collisions at c.m. energies $\sqrt{s}=200 \mathrm{GeV}, 546 \mathrm{GeV}$ and $900 \mathrm{GeV}$ [15], by the UA1 Collaboration for $\bar{p} p$ collisions at $\sqrt{s}=546 \mathrm{GeV}$ [16], by the CDF and the E735 Collaborations for $\bar{p} p$ collisions at $\sqrt{s}=1800 \mathrm{GeV}[17,18]$, and more recent CERN LHC data obtained in $p p$ interactions at $\sqrt{s}=900 \mathrm{GeV}, 2360 \mathrm{GeV}$, and $7 \mathrm{TeV}$ by the ALICE Collaboration [19] and by the CMS Collaboration [20].

Pseudorapidity spectra of charged particles in elastic and non-single diffraction (NSD) protonproton interactions at $200 \mathrm{GeV} \leq \sqrt{s} \leq 14 \mathrm{TeV}$ are shown in Fig. 1(a). Available experimental data are plotted onto the model calculations as well. The hypothesis of the so-called Feynman scaling [21] postulates that the density of produced charged particles at midrapidity $d N^{c h} / d \eta$ should be saturated somewhere at very high energies. This scaling regime is obviously not reached yet. Moreover, at LHC energies $d N^{c h} /\left.d \eta\right|_{\eta=0}$ demonstrates a non-linear rise with $\ln s$, as suggested by the saturation of the Froissart bound. For $p p$ collisions at top LHC energy $\sqrt{s}=14 \mathrm{TeV}$ the QGSM predicts $d N_{\text {inel }} /\left.d \eta\right|_{\eta=0}=6.1, d N_{N S D} /\left.d \eta\right|_{\eta=0}=7.0$, respectively. The power-law fit to particle densities at midrapidity suggested by the theory of Color Glass Condensate (CGC) in [22] yields the following energy dependence

$$
\left.\frac{d N_{N S D}}{d \eta}\right|_{\eta=0}(s)=0.77 E^{0.23},
$$

where $E=\sqrt{s} / 2$. Thus, the power-law dependence describing the average transverse momentum of charged hadrons as a function of $\sqrt{s}$ should have a form $\left\langle p_{T}\right\rangle=A+B E^{0.23 / 2}$, where $A$ and $B$ are constants to be determined from the fit. The transverse momentum distributions of charged hadrons in NSD collisions at energies in question are shown in Fig. 1(b) together with the experimental data. The agreement between the model results and the data is pretty good. Predictions for $\sqrt{s}=14 \mathrm{TeV}$ are plotted as well. Indeed, the average $p_{T}$ of the generated spectra can be well reproduced by the power law

$$
\left\langle p_{T}\right\rangle=0.27+0.212 E^{0.115}
$$

Another scaling relation related to Feynman scaling is the extended longitudinal scaling (ELS) [23] exhibited by the slopes of (pseudo)rapidity spectra. In the QGSM these slopes are identical 
(a)
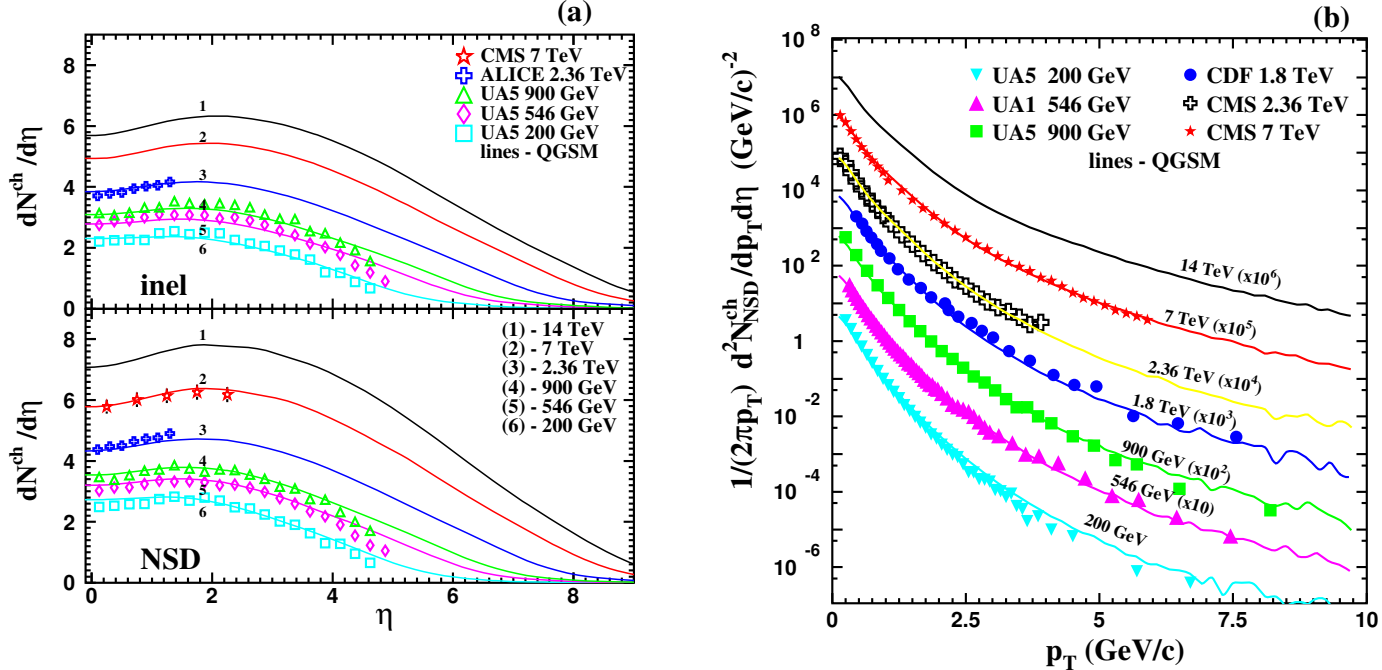

Figure 1. (a): Pseudorapidity spectra for charged particles in inelastic and NSD $p p$ collisions at $200 \mathrm{GeV} \leq$ $\sqrt{s} \leq 14 \mathrm{TeV}$. (b): transverse momentum distribution of the invariant cross section in NSD $p p$ collisions in the same energy range.

in the fragmentation region $y_{\text {beam }} \geq-2.5$ as shown in Fig. 2, where the distributions $\frac{1}{\sigma_{N S D}} \frac{d \sigma_{N S D}}{d y}$ are expressed as functions $y-y_{\max }$. QGSM indicates that the ELS remains certainly valid at LHC. Obtained result contradicts to the prediction based on the statistical thermal model [24]. The latter fits the measured rapidity distributions to the Gaussian, extracts the widths of the Gaussians and implements the energy dependence of the obtained widths to simulate the rapidity spectra at LHC. The extrapolated distribution was found to be much narrower [24] compared to that presented in Fig. 2. Further LHC measurements of $p p$ collisions in the fragmentation regions are needed to resolve this obvious discrepancy. Note, that experimentally the extended longitudinal scaling was found to hold to $10 \%$ in a broad energy range from $\sqrt{s}=30.8 \mathrm{GeV}$ to $900 \mathrm{GeV}$ [15].

The extended longitudinal scaling in the QGSM emerges merely due to short range correlations in rapidity space. The correlation function of particle $i$ and particle $j$, produced as a result of a string fragmentation, drops exponentially with rising rapidity difference

$$
\begin{aligned}
C\left(y_{i}, y_{j}\right) & =\frac{d^{2} \sigma}{\sigma_{\text {inel }} d y_{i} d y_{j}}-\frac{d \sigma}{\sigma_{\text {inel }} d y_{i}} \frac{d \sigma}{\sigma_{\text {inel }} d y_{j}} \\
& \propto \exp \left[-\lambda\left(y_{i}-y_{j}\right)\right],
\end{aligned}
$$

and, therefore, the particles with large rapidity difference are uncorrelated. Consider now the inclusive process $1+2 \rightarrow i+X$. Its single particle inclusive cross section

$$
f_{i} \equiv E \frac{d^{3} \sigma_{i}}{d^{3} p}=\frac{d^{2} \sigma\left(y_{1}-y_{i}, y_{i}-y_{2}, p_{i T}^{2}\right)}{d y_{i} d^{2} p_{i T}}
$$

becomes independent of $y_{i}-y_{2}$ at sufficiently high collision energy in the fragmentation region of particle 1 , provided $y_{1}-y_{i} \approx 1$ and $y_{i}-y_{2} \approx y_{1}-y_{2} \gg 1$. Thus, the inclusive densities $n_{i} \equiv f_{i} / \sigma_{\text {inel }}$ 

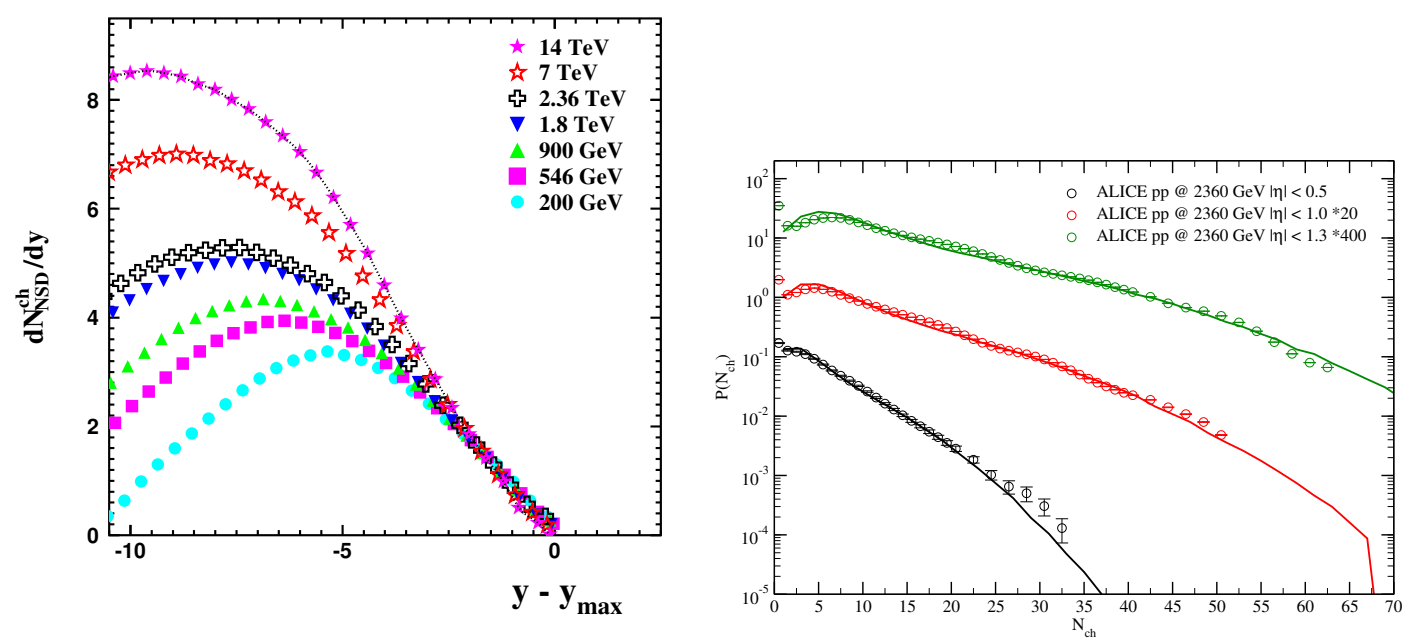

Figure 2. The distributions $\frac{1}{\sigma_{N S D}} \frac{d \sigma_{N S D}}{d y}$ of charged particles as functions of rapidity difference $y-y_{\max }$ obtained in QGSM for $p p$ collisions at all energies in question.

Figure 3. Charged-particle multiplicity distributions in $|\eta|<0.5,|\eta|<1.0$ and $|\eta|<1.3$ intervals, obtained in QGSM for $p p$ collisions at $\sqrt{s}=2360 \mathrm{GeV}$. Open symbols present the ALICE data [19].

are determined by only two variables

$$
n_{i}=\phi\left(y_{1}-y_{i}, p_{i T}^{2}\right)
$$

Another scaling dependence is known as Koba-Nielsen-Olesen (KNO) scaling [25]. It claims that at $\sqrt{s} \rightarrow \infty$ the normalized multiplicity distribution just scales up as $\ln s$ or, equivalently, that

$$
\frac{\langle n\rangle \sigma_{n}}{\Sigma \sigma_{n}}=\Psi\left(\frac{n}{\langle n\rangle}\right),
$$

with $\sigma_{n}$ being the partial cross section for $n$-particle production, $\langle n\rangle$ - the average multiplicity and $\Psi(n /\langle n\rangle)$ - energy independent function. KNO-scaling was found to hold up to ISR energies, $\sqrt{s} \leq 62 \mathrm{GeV}$. Violation of the KNO-scaling was predicted within the QGSM in [1]. Later on the violation was observed experimentally by the UA5 and UA1 collaborations in $\bar{p} p$ collisions at $\sqrt{s}=546 \mathrm{GeV}$ in the full phase space [15]. The rapidity range is crucial for this study. In very central pseudorapidity window $|\eta|<0.5$ the KNO-scaling is still maintained at $\sqrt{s}=2.36 \mathrm{TeV}$ [19], as seen in Fig. 3, whereas already the UA5 Collaboration observed progressive violation of the scaling with increasing $\eta$ intervals at much lower energies. For a bit broader midrapidity intervals at LHC a peak at low multiplicities seems to appear, see Fig. 3. The origin of this phenomenon in the model is the following. At ultrarelativistic energies the main contribution to particle multiplicity comes from the cut-Pomerons, and each cut results to formation of two strings. Short range correlations inside a single string lead to a Poisson-like multiplicity distribution of produced secondaries. At energies below $100 \mathrm{GeV}$ the multi-string (or chain) processes are not very abundant and invariant masses of the strings are not very large. Therefore, different contributions to particle multiplicity overlap strongly, 

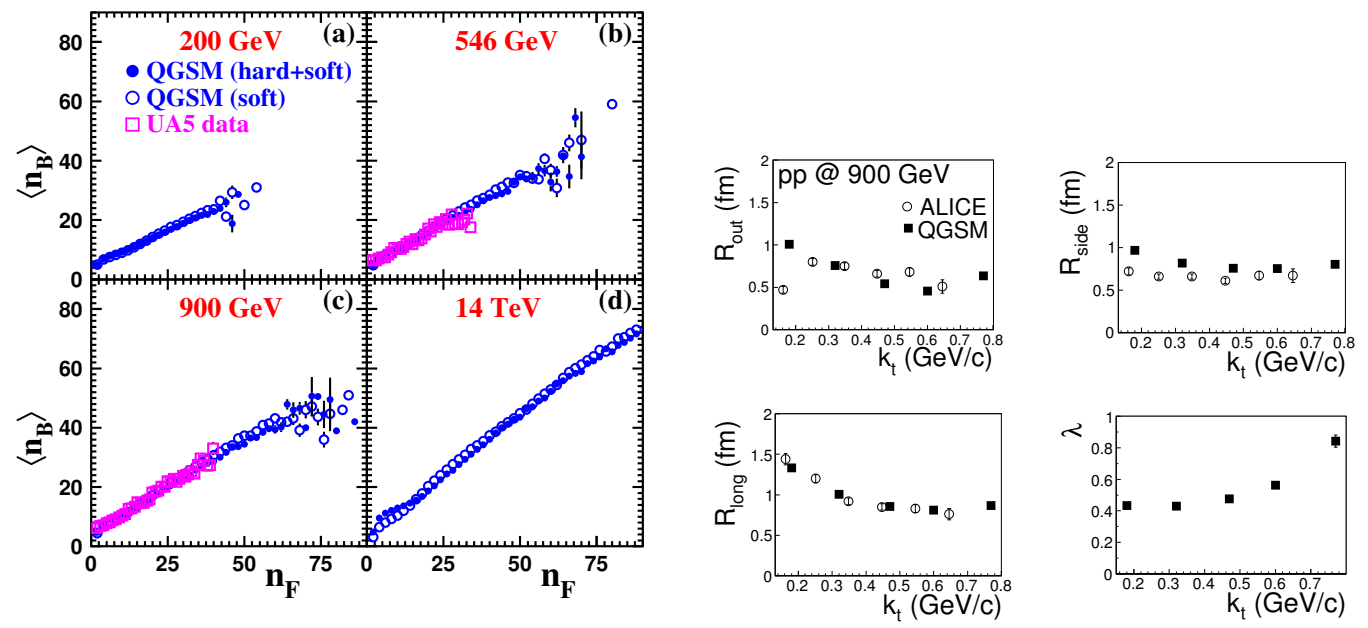

Figure 4. Backward-forward multiplicity correlations Figure 5. Three-dimensional $\pi^{+} \pi^{+}$correlation radii as $\left\langle n_{B}\left(n_{F}\right)\right\rangle$ for $0 \leq|\eta| \leq 4$ in NSD $p p$ interactions at $\sqrt{s}=$ functions of $k_{T}$ in $p p$ collisions at $\sqrt{s}=900 \mathrm{GeV}$ for $200 \mathrm{GeV}$ (a), $546 \mathrm{GeV}$ (b), $900 \mathrm{GeV}$ (c) and $14 \mathrm{TeV}$ (d). minimum bias events. Open circles denote ALICE exOpen circles denote contributions of soft processes, full perimental data, full squares present QGSM calculasymbols are for all processes. Data are from [15]. tions.

and the KNO-scaling is nearly fulfilled. With rising $\sqrt{s}$ the number of strings increases as $\left(s / s_{0}\right)^{\Delta}$ and their invariant masses increase as well. This leads to enhancement of high multiplicities, deviation of the multiplicity distribution from the Poisson-like behavior and violation of KNO-scaling $[1,6]$.

\subsection{Long-range and femtoscopy correlations}

Long-range correlations between charged particles emitted in forward (F) and backward (B) hemispheres were first observed in [26]. The strength of the correlations is defined as

$$
b=\frac{\left\langle\left(n_{F}-\left\langle n_{F}\right\rangle\right)\left(n_{B}-\left\langle n_{B}\right\rangle\right)\right\rangle}{\left[\left\langle\left(n_{F}-\left\langle n_{F}\right\rangle\right)^{2}\right\rangle\left\langle\left(n_{B}-\left\langle n_{B}\right\rangle\right)^{2}\right\rangle\right]^{1 / 2}},
$$

where $n_{F}$ and $n_{B}$ represent multiplicities of charged particles in forward and backward hemispheres, respectively. In Fig. 5 we show the dependence of the mean charged-particle multiplicity in the backward hemisphere $\left\langle n_{B}\right\rangle$, measured in the range $-4 \leq \eta \leq 0$, on the multiplicity in the forward hemisphere $n_{F}$ for the symmetric range $0 \leq \eta \leq 4$ at four energies in question. Comparison with experimental data at $\sqrt{s}=546 \mathrm{GeV}$ and $900 \mathrm{GeV}$ shows a good agreement between the model results and the data. The dependence looks pretty linear

$$
\left\langle n_{B}\left(n_{F}\right)\right\rangle=a+b n_{F},
$$

and its slope $b$ increases with energy. In the QGSM the rise of the strength of correlations is linked to increase of number of Pomerons, i.e. strings, with energy in the aforementioned pseudorapidity 
range. As one can see from Fig. 4, the correlations between $\left\langle n_{B}\right\rangle$ and $n_{F}$ are fully determined by soft processes.

The momentum correlations are usually studied by means of two-particle correlation functions defined as a ratio of the two-particle distributions from the same event to the reference ones. Generally, the correlations are measured as a function of pair relative momenta four vector $q$ or rather its invariant form $q_{i n v}=\sqrt{q_{0}^{2}-|q|^{2}}$.

The 3D correlation analysis can provide information about both the form of the emitting source and the duration of the emission [27, 28]. Here the momentum correlation functions are analyzed in terms of the out, side and longitudinal components of the relative momentum vector $\mathbf{q}=\left\{q_{\text {out }}, q_{\text {side }}, q_{\text {long }}\right\}$, where $q_{\text {out }}$ and $q_{\text {side }}$ denote the transverse components of the vector $\mathbf{q}$, and the direction of $q_{\text {out }}$ is parallel to the transverse component of the pair three-momentum. The corresponding correlation widths are usually parametrized in terms of the Gaussian correlation radii

$$
C F\left(p_{1}, p_{2}\right)=1+\lambda \exp \left(-R_{\text {out }}^{2} q_{\text {out }}^{2}-R_{\text {side }}^{2} q_{\text {side }}^{2}-R_{\text {long }}^{2} q_{\text {long }}^{2}\right) .
$$

The extracted $R_{i}$ as functions of average pair transverse momentum $k_{T}=\left|\overrightarrow{p_{t, 1}}+\overrightarrow{p_{t, 2}}\right| / 2$ are presented in Fig. 5 for the low multiplicity bin in $p p$ interactions at $\sqrt{s}=900 \mathrm{GeV}$. One can see that the QGSM points are rather close to the ALICE experimental ones [29]. Formally, this implies significant reduction of the formation time with increasing energy [30]. Recently, however, it was shown [31] that quantum corrections to pion interferometry results in $p p$ collisions at LHC energies could drastically improve the agreement between the model results and the data.

\section{Conclusions}

We apply the quark-gluon string model, based on Reggeon Field Theory, for the description of protonproton collisions at ultrarelativistic energies. It is shown that simulations of pseudorapidity, transverse momentum and multiplicity spectra of secondaries are in a good agreement with the corresponding experimental data obtained in $\bar{p} p$ and $p p$ collisions at Tevatron and at CERN energies. Predictions are made for $p p$ interactions at top LHC energy $\sqrt{s}=14 \mathrm{TeV}$.

Several scaling properties observed in particle production at relativistic energies have been examined. QGSM favors violation of Feynman scaling in the central rapidity region. Extended longitudinal scaling is shown to hold at LHC, whereas further violation of the KNO-scaling in multiplicity distributions is demonstrated. The origin of both conservation and violation of the scaling trends is traced to short range correlations of particles in the strings and interplay between the multi-Pomeron processes at ultra-relativistic energies.

\section{References}

[1] A.B. Kaidalov, Phys. Lett. B 116, 459 (1982);

A.B. Kaidalov and K.A. Ter-Martirosyan, Phys. Lett. B 117, 247 (1982).

[2] A. Capella, U. Sukhatme, C.-I. Tan, and J. Tran Thanh Van, Phys. Rep. 236, 225 (1994).

[3] V.N. Gribov, Sov. Phys. JETP 26, 414 (1968).

[4] G. t'Hooft, Nucl. Phys. B 72, 461 (1974).

[5] G. Veneziano, Phys. Lett. B 52, 220 (1974).

[6] N.S. Amelin and L.V. Bravina, Sov. J. Nucl. Phys. 51, 133 (1990).

[7] L. V. Bravina et al., Phys. Rev. C 60, 044905 (1999);

E. E. Zabrodin et al., Phys. Lett. B 508, 184 (2001). 
[8] G. Burau et al., Phys. Rev. C 71, 054905 (2005);

J. Bleibel et al., Phys. Lett. B 659, 520 (2008).

[9] V. Abramovskii, V. Gribov, O. Kancheli, Sov. J. Nucl. Phys. 18, 308 (1974).

[10] A. B. Kaidalov, Surveys in High Energy Phys. 13, 265 (1999).

[11] M. Baker, K. A. Ter-Martirosyan, Phys. Rep. 28C, 1 (1976).

[12] N.S. Amelin, E.F. Staubo, and L.P. Csernai, Phys. Rev. D 46, 4873 (1992).

[13] R. D. Field, R. P. Feynman, Nucl. Phys. B 136, 1 (1978).

[14] A. Bialas, M. Gyulassy, Nucl. Phys. B 291, 793 (1987).

[15] G. J. Alner et al. (UA5 Collab.), Phys. Rep. 154, 247 (1987).

[16] G. Arnison et al. (UA1 Collab.), Phys. Lett. B 118, 167 (1982);

C. Albajar et al. (UA1 Collab.), Nucl. Phys. B 335, 261 (1990).

[17] F. Abe et al. (CDF Collab.), Phys. Rev. Lett. 61, 1819 (1988); Phys. Rev. D 41, R2330 (1990).

[18] T. Alexopoulos et al. (E735 Collab.), Phys. Rev. D 48, 984 (1993).

[19] ALICE Collaboration (K. Aamodt et al.), Eur. Phys. J. C 68, 89 (2010);

Eur. Phys. J. C 68, 345 (2010);

Phys. Lett. B 693, 53 (2010).

[20] CMS Collaboration (K. Khachatryan et al.), JHEP 1002, 041 (2010):

Phys. Rev. Lett. 105, 022002 (2010).

[21] R. Feynman, Phys. Rev. Lett. 23, 1415 (1969);

R. Feynman, Photon-hadron interactions (Benjamin, 1972).

[22] L. McLerran and M. Praszalowicz, Acta Phys. Polon. B 41, 1917 (2010).

[23] G.J. Alner et al. (UA5 Collab.), Z. Phys. C 33, 1 (1986).

[24] J. Cleymans, J. Strümpfer, and L. Turko, Phys. Rev. C 78, 017901 (2008).

[25] Z. Koba, H.B. Nielsen, and P. Olesen, Nucl. Phys. B 40, 317 (1972).

[26] C.J. Alner et al. (UA5 Collab.), Nucl. Phys. B 291, 445 (1987).

[27] M.I. Podgoretsky, Fiz. Elem. Chast. Atom. Yadra 20, 628 (1989) (in Russian).

[28] R. Lednicky, Phys. Atom. Nucl. 67, 72 (2004).

[29] ALICE Collaboration (K. Aamodt et al.), Phys. Rev. D 82, 052001 (2010).

[30] M.S. Nilsson et al., Phys. Rev. D 84, 054006 (2011).

[31] V.M. Shapoval et al., Phys. Lett. B 725, 139 (2013). 